\title{
Visual layout modulates Fitts's law: The importance of first and last positions
}

\author{
Jay Pratt \\ University of Toronto, Toronto, Ontario, Canada \\ Jos J.ADAM \\ University of Maastricht, Maastricht, The Netherlands \\ AND \\ MARTIN H. Fischer \\ University of Dundee, Dundee, Scotland
}

\begin{abstract}
When a target in the last position of a structured visual array is aimed for, movement times (MTs) are shorter than predicted by Fitts's law (Adam, Mol, Pratt, \& Fischer, 2006). That study, however, confounded relative target position with absolute target location. To determine whether target position does, indeed, produce changes in the speed-accuracy trade-off function, the present experiment manipulated relative target position (e.g., first or last) independently of absolute target location (e.g., nearest or farthest). This was accomplished by presenting connected placeholders at three adjacent locations from a set of five possible locations (i.e., the middle location could be the first, middle, or last placeholder position in an array). The results of a speeded manual-pointing task showed that relative position is important for Fitts's law; when absolute location was held constant, shorter MTs were found for last-position than for middle-position targets. In addition, a similar effect was found for first-position targets. These results suggest that Fitts's law holds within, but not between, relative target positions in visible structured arrays.
\end{abstract}

Perhaps no aspect of our motor system pervades our daily lives more than the balance we strike, for virtually every action, between speed and accuracy. Indeed, all of us have some intuitive awareness that as we move faster, our spatial accuracy decreases, and as the accuracy requirements increase, we must slow down our movements. For example, inserting a key into a keyhole is a task that requires considerable precision in terms of motor planning and production. This task is accomplished quite easily when there are no time constraints. However, inserting the same key into the same keyhole while rushing to answer a ringing phone is a much more difficult task.

The study of speed-accuracy trade-offs in the human motor system goes back to Woodworth (1899). It was the seminal work by Paul Fitts (1954), however, that prompted the modern approach to the study of motor control. In his most famous experiment, participants moved their hand between targets that varied in terms of their width (i.e., how big the targets were) and amplitude (i.e., the distance between the two targets). He found that the movement times (MTs) of these movements could be accounted for by the equation MT $=a+b\left[\log _{2}(2 A / W)\right]$, where $A$ is target amplitude and $W$ is target width, with $a$ and $b$ being empirical constants derived from a linear approximation to the data. For the crucial portion of the equation, $\log _{2}(2 A / W)$, Fitts coined the term index of difficulty (ID).

The equation that predicts MT from target width and amplitude has proven to hold under such a wide range of tasks and situations that it has become known as Fitts's law. It has been the focus of more than 200 published studies (nearly 150 of which have been published in the last 10 years). The equation has been shown to hold with prehension movements (e.g., Bootsma, Marteniuk, MacKenzie, \& Zaal, 1994), mouse cursor movements (e.g., Lin, Radwin, \& Vanderheiden, 1992), rotational movements (e.g., Abrams, Meyer, \& Kornblum, 1990), head movements (e.g., Jagacinksi \& Monk, 1985), and foot movements (e.g., Hoffmann, 1991). The equation holds underwater (Kerr, 1973), in near zero-gravity environments (Jungling, Bock, \& Girgenrath, 2002), with microscopic targets (Langolf, Chaffin, \& Foulke, 1976), with imaginary movements (Sirigu et al., 1996), and with targets that have illusory IDs (e.g., Fischer, 2001). Although there have been several refinements to the original equation (for a review, see Plamondon \& Alimi, 1997), Fitts's law continues to account for a vast amount of data and can arguably be considered the most successful and well-researched law in cognitive psychology. 

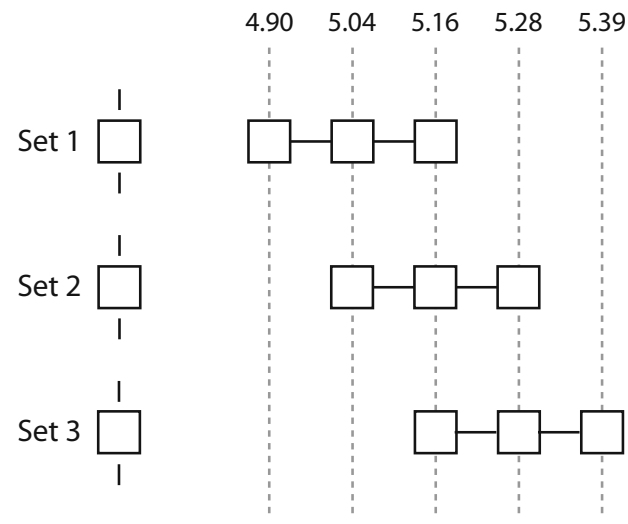

Figure 1. The three sets of placeholder arrays used in the experiment. For each set, the home position appears to the left, and the three connected placeholders appear to the right. The $\log _{2}$ index of difficulty for each location is given at the top of the figure, and the dashed lines are for expository purposes only and did not appear during the experiment. The target (not shown here) consisted of a green square that completely filled in one of the placeholders.

Recently, we have shown that a simple perceptual manipulation can produce a striking modulation of Fitts's law (Adam, Mol, Pratt, \& Fischer, 2006). Using a touch screen, participants made guided pointing movements from a home position to a target that could appear in one of seven horizontally aligned locations to the right of the home position. Because target width remained constant, ID varied as a function of target amplitude. In the control condition, only the home position and the target position for each trial were shown, and the MTs for all seven targets conformed to Fitts's law. In the experimental condition, placeholder boxes, which surrounded all seven locations, were presented prior to the appearance of each target. In this condition, MTs for the first six locations once again conformed to Fitts's law, but the MT to the last location was actually shorter than that to the secondto-last location. This exception to Fitts's law, for targets at the end location of a visible structured array of placeholders, was replicated in a second experiment with five vertically aligned placeholders. These findings indicate that final locations in arrays have special status, perhaps acting as spatial anchoring points. Such anchoring points suggest the operation of an environment-centered frame of reference, which further suggests that Fitts's law may be limited to tasks that rely on egocentric information, whereas the visual control of limb movements across saliently structured arrays also may use allocentric spatial information.

One potential weakness in the design used by Adam et al. (2006) was that absolute target location was confounded with relative target position. Thus, the violation of Fitts's law was based on the finding that the MTs to the last location in the array were shorter than the expected MTs to a single target at the same amplitude. Because of this, it remains unclear whether the relative position of the target (i.e., last target in an array of targets) or the absolute location of the target (i.e., furthest target from the home position) is responsible for the change in the speedaccuracy trade-off function. To address this confound of allocentric and egocentric position, in the present experiment, the basic methodology of Adam et al.'s study was used, with two important changes (see Figure 1). First, there were five possible target locations, but on any given trial, placeholders would appear only at three adjacent locations. In other words, the placeholders could appear at locations $1-2-3,2-3-4$, or 3-4-5. This allowed for the comparison of different array positions (first, middle, and last) while holding target location constant. For example, a target at Spatial Location 3 would be at the last position of the 1-2-3 array, the middle position of the 2-3-4 array, or the first position of the 3-4-5 array. Second, two lines connected the three placeholders in order to produce a clearly structured visible array. Such lines produce element connectedness, an extremely powerful grouping principle (Palmer \& Rock, 1994) that helps to highlight the first and last locations in the arrays.

By unconfounding the relative position and the absolute locations of the targets, it is also possible to further examine another interesting aspect of the Adam et al. (2006) data: Whereas movements to targets at the last array position showed marked deviations from Fitts's law, the same did not occur with targets at the first array position. Yet the first array position is as perceptually salient as the last array position, and if allocentric spatial information supported the shorter MTs to the last array position, this should also aid movements to the first array position. However, the target in the first position of the array was always nearest the home position, just as the target in the last position of the array was always furthest from the home position. Thus, the ID for the nearest target was always the lowest within the range, and short MTs were expected for pointing responses to this target. This confound may have prevented the array effect from emerging for first-position targets in our earlier study.

The prediction regarding MTs to targets at the last array positions, in comparison with those to targets at the middle array positions, follows directly from Adam et al. (2006); movements should be faster for targets at last positions than for those at middle positions when these targets occur at the same spatial location. In other words, for movements with identical IDs, MTs will be affected by the relative position of the target in an array of potential targets. If this first prediction holds, we suspect that a similar pattern of results will be found for first positions, because such positions share a unique status similar to that for the end positions.

\section{METHOD}

\section{Participants}

Twenty-one undergraduate students from the University of Toronto participated in the study in exchange for class credit. All were right-handed and had normal or corrected-to-normal vision.

\section{Apparatus and Procedure}

The experiment was conducted in a dimly lit, sound-attenuated testing room on a 21-in. ELO touchscreen connected to a Pentium computer. The touchscreen was angled backward $\left(15^{\circ}\right)$ to make 
movements more comfortable, and the participants were allowed to sit or stand, whichever they preferred, during the session.

Each trial began with the presentation of the home position and three connected placeholder boxes to the right (see Figure 1). To help differentiate the home position box from the placeholder boxes, the home box had small vertical lines located just above and below it. The background was black, and all the boxes were white and $11.2 \mathrm{~mm}$ (25 pixels) square. When the participant placed the index finger of his or her preferred hand within the home position box, the small vertical lines above and below the box disappeared to warn the participant of the upcoming target. Then, $500 \mathrm{msec}$ after the offset of the lines, a green square filled one of the three placeholder boxes. The participant was instructed to point to (i.e., to move to and hit) the target as quickly and accurately as possible. Once the movement was completed, the display was blanked, and a 1,000-msec delay occurred before the start of the next trial. If the participant did not land inside the target placeholder, a brief error tone was presented, and the trial was tagged as an error and was not included in the analyses.

Reaction time (RT) was the time from target onset until the participant's index finger lifted off from the start position. This measure is an established indicator of movement planning (e.g., Henry \& Rogers, 1960; Rosenbaum, 1980). MT was the time from this liftoff until the next touch-down occurred. This measure is an established indicator of online control, as was discussed above. Both measures were recorded to the nearest millisecond. Movement amplitude was the Euclidean distance between the coordinates of a liftoff and the subsequent touchdown, measured in pixels and converted to millimeters. Effective target width was calculated as 4.133 times the participant's standard deviation of horizontal touchdown coordinates (e.g., Welford, 1968, p. 148). Errors (i.e., landing outside the target boundary) were defined as landing positions that were outside the horizontal or vertical boundary of the target.

As was noted above, a key aspect of the study was that there were five target locations (center-to-center distances from the home position: $168,185,202,219$, and $236 \mathrm{~mm}$, or $383,421,460,498$, and 537 pixels, respectively, corresponding to ID values of 4.90, 5.04, $5.16,5.28$, and 5.39 bits, respectively), but the three placeholders could appear only in three adjacent locations. Each participant completed 270 trials, 90 from each set of three placeholders. Within each set, there were 30 targets at each position (first, middle, and last). The set of placeholders was randomized across the session, with the target position randomized across each placeholder set.

\section{RESULTS}

For the purpose of the analysis, position will refer to the first, middle, and last positions within a set of three placeholders, set will refer to the position of the three placeholders across the five target locations (from left to right, Set 1, Set 2, and Set 3), and location will refer to the spatial location of the target with a given ID $(4.9,5.05,5.16,5.28$, and 5.39 bits). A 3 (position) $\times 3$ (set) ANOVA determined the effect of array position and set on MTs, RTs, error rates, movement amplitudes, and effective target widths, regardless of location. The mean MTs are shown in Figure 2. A main effect for set was found $[F(2,40)=27.9, p<.001]$, as MTs increased from Set 1 to Set 3 (because, overall, ID increased from Set 1 to Set 3). A main effect for position was also found $[F(2,40)=64.6, p<.001]$, with movements to targets at first $(390 \mathrm{msec})$ and last positions (408 msec) having shorter MTs than did those to middle positions $(415 \mathrm{msec})$. The interaction was not significant $[F(4,80)<1]$. It is worth noting that if MT was strictly determined by absolute spatial location, a main effect of posi-

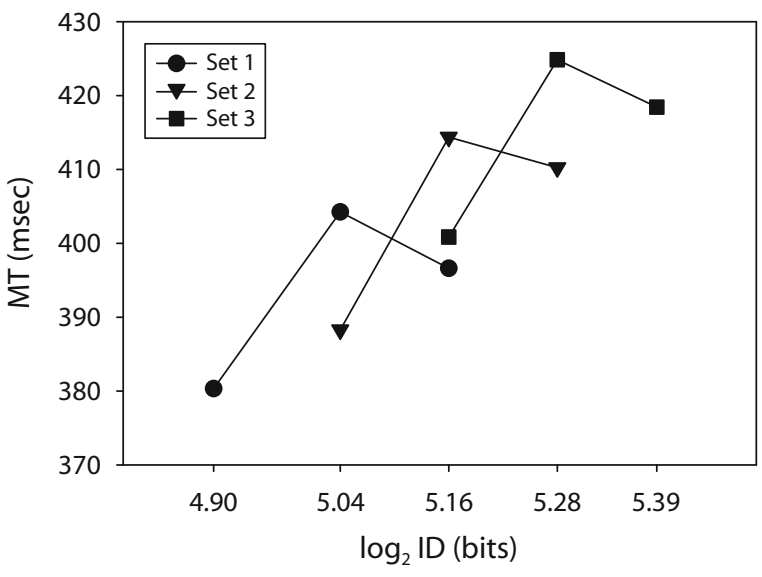

Figure 2. The mean movement times (MTs) for movements to targets at each of the three positions, for each of the three sets of placeholder arrays. ID, index of difficulty.

tion would also be found (because, for example, last positions have the highest average IDs). To examine differences in the speed-accuracy trade-off function across relative target positions, more specific comparisons are required.

To examine the central issue of this article, whether MTs to first and last positions are shorter than MTs to middle positions when absolute spatial location is held constant, four planned $t$ tests (two-tailed) were conducted. Their results unanimously confirmed the special status of both first and last positions, relative to middle positions, in a set. At the 5.04-bit location, MTs were shorter for the first position of Set 2 than for the middle position of Set $1[t(20)=4.1, p<.001]$. At the 5.16-bit location, MTs were shorter for the last position of Set 1 than for the middle position of Set $2[t(20)=5.6, p<.001]$ and for the first position of Set 3 than for the middle position of Set $2[t(20)=2.9, p<.01]$. Finally, at the 5.28-bit location, MTs were shorter for the last position of Set 2 than for the middle position of Set $3[t(20)=3.7, p<.001]$.

To determine whether the effects in MT were due to differential movement amplitudes, a 3 (position) $\times 3$ (set) ANOVA was conducted on mean movement amplitudes of the responses (i.e., the actual distances traveled by the limb; see Table 1). As one would expect, there were main effects for both set $[F(2,40)=18,022, p<.001]$ and position $[F(2,40)=7,442, p<.001]$. These analyses indicate that movement amplitudes were shortest for Set 1 and largest for Set 3 and were shortest for first-position targets and largest for last-position targets. The interaction between the factors was not significant $[F(2,40)<$ $1.4, p>.25]$. Although this indicates that the MT results were not due to a change in movement amplitude, a further test was conducted using a similar set of planned $t$ tests similar to those for the MT analysis, but on the amplitudes of movements to same-location targets. Mimicking the results of the previous ANOVA, no significant effects [all $t \mathrm{~s}(20)<1.3$, all $p \mathrm{~s}>$.30] were found for movement amplitudes toward different position targets at the same location. Thus, the shorter MTs for first- and last-position 
Table 1

Mean Movement Amplitude, End Point Variability (Standard Deviation), Reaction Time (RT), Effective Target Width $\left(W_{\mathrm{e}}\right)$, and Errors for the First, Middle, and Last Positions for the Three Array Sets

\begin{tabular}{lccccc}
\hline Position & $\begin{array}{c}\text { Amplitude } \\
(\mathrm{msec})\end{array}$ & $\begin{array}{c}\text { Variability } \\
(\mathrm{msec})\end{array}$ & $\begin{array}{c}\mathrm{RT} \\
(\mathrm{msec})\end{array}$ & $\begin{array}{c}W_{\mathrm{e}} \\
(\mathrm{msec})\end{array}$ & $\begin{array}{c}\text { Errors } \\
(\%)\end{array}$ \\
\hline & \multicolumn{5}{c}{ Set 1} \\
First & 173 & 1.26 & 338 & 13.08 & 13.1 \\
Middle & 189 & 1.60 & 339 & 12.52 & 12.5 \\
Last & 207 & 1.37 & 332 & 13.85 & 10.4 \\
& & Set 2 & & & \\
First & 190 & 2.28 & 330 & 13.51 & 11.8 \\
Middle & 207 & 1.32 & 337 & 13.55 & 12.0 \\
Last & 225 & 1.39 & 328 & 14.54 & 11.1 \\
& & Set 3 & & & \\
First & 206 & 1.87 & 325 & 14.07 & 11.4 \\
Middle & 223 & 1.45 & 334 & 13.77 & 12.2 \\
Last & 240 & 1.50 & 336 & 13.95 & 13.2 \\
\hline
\end{tabular}

targets, as compared with middle-position targets, were not due to shorter amplitude movements.

A series of 3 (set) $\times 3$ (position) ANOVAs were also conducted on the mean RTs, effective target widths, and number of error trials (see Table 1). For RTs, no main effects $\left(F_{\mathrm{S}}<1.1\right)$ and no set $\times$ position interaction $(F<1)$ were found. The same was true for effective target widths $\left(F_{\mathrm{S}}<1.2, p \mathrm{~s}>.20\right)$ and the number of error trials $\left(F_{\mathbf{S}}<1\right)$. Thus, the differences in MTs were not due to more variable movements, greater errors, shorter movements, or shorter RTs being associated with targets at the first and last positions.

As was noted earlier, Fitts's law describes a logarithmic speed-accuracy trade-off function, so that when MT is plotted against ID, the resulting plots are predicted to be linear in nature. To examine whether Fitts's law holds within positions, separate linear regressions were conducted on each of the three positions across the three sets (i.e., first positions across Set 1, Set 2, and Set 3; middle positions across Set 1, Set 2 and Set 3; and last positions across Set 1, Set 2, and Set 3). The results show that Fitts's law holds for first-position targets $\left(r^{2}=.969\right)$, middleposition targets $\left(r^{2}=.999\right)$, and last-position targets $\left(r^{2}=\right.$ .987). Moreover, combining first- and last-position targets produced a strong Fitts's law function $\left(r^{2}=.985\right)$, whereas combining first-, middle-, and last-position targets resulted in much poorer fit $\left(r^{2}=.722\right)$. When these two possible linear fits (first and last vs. global fit) were determined separately for each individual participant's data set, a paired $t$ test on the resulting (Fisher's $Z$ transformed) $r^{2}$ values showed a significantly reduced fit for the global approach, as compared with the first-and-last approach $[t(20)=4.36, p<.001]$.

\section{DISCUSSION}

In a recent study, Adam et al. (2006) showed that structured perceptual arrays can modulate Fitts's law. More specifically, they found that the movements made to targets at the last position in a set of placeholder boxes produced MTs that were shorter than would be predicted from the MTs to the other target positions. However, the Adam et al. study confounded the relative position and the absolute location of the target. To overcome this confound and to further examine this modulation of Fitts's law, the present study was conducted.

The most important finding from the present study was that the MT advantage discovered by Adam et al. (2006) was replicated even when target location was controlled for. More specifically, for all three sets, MTs to targets at the final positions were equal to, or shorter than, MTs to targets at middle positions (see Figure 2). This pattern of results occurred even when final-position targets occurred at the same absolute spatial locations as middleposition targets. In other words, when spatial location was held constant, the results still replicated the modulation of Fitts's law found by Adam et al. Thus, the relative position of a target in a structured perceptual array can affect the speed-accuracy trade-off function described by Fitts's law.

The second important aspect of the data is the finding that first-position targets are also capable of modulating Fitts's law. The case here is not as easily made as with the last positions, because first locations, by definition, have lower IDs than do middle positions and, thus, shorter MTs are expected. However, the exception can be seen by comparing MTs for different array positions that were directed toward targets at the same spatial location. The best example of this comes from examining MTs to targets at the last position in Set 1, the middle position in Set 2, and the first position in Set 3. The MTs to the first position in Set 3 were shorter than the MTs to the middle position in Set 2 and very similar to the MTs to the last position in Set 1. Assuming that the MTs for the last position in Set 1 reflect an exception to Fitts's law (as indicated by both the present data and those in Adam et al.'s [2006] study), the MTs for the first position in Set 3 appear to reflect a similar exception. In addition, MTs to targets at the first position of Set 2 were also shorter than MTs to the middle position of Set 1 . Thus, the present data clearly show that first positions in a structured perceptual array of placeholders have the same unique status as last positions.

The third aspect of the data that bears mentioning is the strong linear relationship between MT and ID for the first and last positions across the various target distances. This suggests that the first and last positions are not exceptions to Fitts's law but, rather, that Fitts's law interacts with allocentric spatial information, because the logarithmic speed-accuracy trade-off predicted by Fitts's law holds within target positions. In other words, MTs to first-position targets in the three sets conform to Fitts's law, since MTs increase linearly with increases in ID. The same holds for second-position targets across sets, as well as for last-position targets. Thus, the execution of rapid aimed limb movements is very sensitive to allocentric information, such as the relative position of a target location, and within relative positions across various spatial locations, the speed-accuracy trade-off described by Fitts's law holds. 
What is it about first and last positions in an array of placeholders that might produce such dramatic effects on speed-accuracy trade-off functions? We will discuss a perceptual and a visuomotor hypothesis in turn.

A perceptual account of the present findings might suggest that targets in middle positions are harder to perceive than targets in either first or last positions, possibly due to lateral masking from the two adjacent neighbors (e.g., Bouma, 1970). However, at least two observations make this explanation implausible. First, this notion would predict selectively slower response planning for movements to the middle targets, as compared with those to the outer targets. In contrast with this prediction, the absence of RT effects in the present results suggests that our manipulation affected the online control of movement execution, and not movement planning. This aspect of our findings shows that the targets in all the positions were equally perceptible but not equally reachable. And second, in Adam et al.'s (2006) study, MTs for the first six target positions in the visible array condition were virtually identical to the MTs for the corresponding locations in the single-target condition. The mechanism in question, therefore, selectively facilitates responses to the outer elements of a set and does not inhibit responses to central elements within a set. In sum, it is unlikely that poorer perceptual discrimination of middle-position targets, as compared with outer-position ones, accounts for the present results.

A visuomotor interpretation of the present findings might propose that goal-directed movements, once initiated, are easier to control (i.e., need less fine-tuning or adjustment) when aiming for outer positions of an array. In this view, responses toward end positions are easier because the borders of objects act as particularly salient visual landmarks that enable participants to home in on the target with fewer corrections. This notion of a special status of end positions in an array, which may act as natural spatial anchors that facilitate spatial processing, is supported by the well-known finding that a masking stimulus has less effect on location information at end positions than at middle positions of an array of letters (e.g., Mewhort \& Campbell, 1978). In addition, Obhi and Goodale (2005) recently made a similar point by showing that landmarks, once encoded, facilitate pointing movements and reduce error variability. The outcome of this particularly salient spatial coding of end positions is that movements can be made more rapidly to such locations without a corresponding change in accuracy. The visuomotor advantage of landmarks does not occur when targets are presented in blank visual fields, because, in this situation, the landmarks (i.e., front and back borders of the single visual object) define the target. This visuomotor hypothesis underlines the impact of the present findings; it now seems unlikely that a single equation will be able to accurately capture all aspects of speed-accuracy tradeoffs, and existing models of motor control will need to be examined, reinterpreted, or possibly replaced.

Regardless of the underlying mechanism, the present findings clearly show the important, and often overlooked, role that relative position of targets plays in speed-accuracy trade-offs. In turn, this provides more evidence for the role of allocentric spatial information in the visual control of action. Although various studies point to the dominance of egocentric spatial information in motor control (e.g., Milner \& Goodale, 1995; Soechting \& Flanders, 1992), the findings in Adam et al. (2006) and in the present study support other recent work (e.g., Elliott \& Meegan, 2004; Keulen, Adam, Fischer, Kuipers, \& Jolles, 2002) that highlights the contribution of allocentric information to online movement control. As is indicated from the present results, the influence of allocentric information can be so profound that Fitts's law occurs for targets that vary in ID within, but not across, positions in perceptually structured arrays.

\section{AUTHOR NOTE}

This work was supported by an NSERC grant to J.P. The authors thank Howie Zelaznik, Andrew Slifkin, and an anonymous reviewer for their helpful comments. Correspondence regarding this article should be addressed to J. Pratt, Department of Psychology, University of Toronto, 100 St. George Street, Toronto, ON, M5S 3G3 Canada (e-mail: pratt@, psych.utoronto.ca).

\section{REFERENCES}

Abrams, R. A., Meyer, D. E., \& Kornblum, S. (1990). Eye-hand coordination: Oculomotor control in rapid aimed limb movements. Journal of Experimental Psychology: Human Perception \& Performance, 16, 248-267.

Adam, J. J., Mol, R., Pratt, J., \& Fischer, M. H. (2006). Moving farther but faster: An exception to Fitts's law. Psychological Science, 17, 794-798.

Bootsma, R. J., Marteniuk, R. G., MacKenzie, C. L., \& ZaAl, F. T. (1994). The speed-accuracy trade-off in manual prehension: Effects of movement amplitude, object size and object width on kinematic characteristics. Experimental Brain Research, 98, 535-541.

Bouma, H. (1970). Interaction effects in parafoveal letter recognition. Nature, 226, 177-178.

Elliott, D., \& Meegan, D. V. (2004). Visual context can influence online control. Behavioral \& Brain Sciences, 27, 33-34.

FisCHER, M. H. (2001). How sensitive is hand transport to illusory context effects? Experimental Brain Research, 136, 224-230.

FitTs, P. M. (1954). The information capacity of the human motor system in controlling the amplitude of movement. Journal of Experimental Psychology, 47, 381-391.

Henry, F. M., \& Rogers, D. E. (1960). Increased response latency for complicated movements and a "memory drum" theory of neuromotor reaction. Research Quarterly, 31, 448-458.

HoFFMANN, E. R. (1991). A comparison of hand and foot movement times. Ergonomics, 34, 397-406.

JAGACINSKI, R. J., \& Monk, D. L. (1985). Fitts' law in two dimensions with hand and head movements. Journal of Motor Behavior, 17, 77-95.

Jungling, S., Bock, O., \& Girgenrath, M. (2002). Speed-accuracy trade-off of grasping movements during microgravity. Aviation, Space, \& Environmental Medicine, 73, 430-435.

KERR, R. (1973). Movement time in an underwater environment. Journal of Motor Behavior, 5, 175-178.

Keulen, R. F., Adam J. J., Fischer, M. H., Kuipers, H., \& Jolles, J. (2002). Selective reaching: Evidence for multiple frames of reference. Journal of Experimental Psychology: Human Perception \& Performance, 28, 515-526.

Langolf, G. D., Chaffin, D. B., \& Foulke, J. A. (1976). An investigation of Fitts' law using a wide range of movement amplitudes. Journal of Motor Behavior, 8, 113-128.

Lin, M. L., Radwin, R. G., \& Vanderheiden, G. C. (1992). Gain effects on performance using a head-controlled computer input device. Ergonomics, 35, 159-175.

Mewhort, D. J. K., \& CAmpbell, A. J. (1978). Processing spatial infor- 
mation and the selective-masking effect. Perception \& Psychophysics, 24, 93-101.

Milner, A. D., \& Goodale, M. A. (1995). The visual brain in action (Oxford Psychology Series, No. 27). New York: Oxford University Press.

OвнI, S. S., \& Goodale, M. A. (2005). Bimanual interference in rapid discrete movements is task specific and occurs at multiple levels of processing. Journal of Neurophysiology, 94, 1861-1868.

PAlmer, S., \& Rock, I. (1994). Rethinking perceptual organization: The role of uniform connectedness. Psychonomic Bulletin \& Review, 1, 29-55.

Plamondon, R., \& Alimi, A. M. (1997). Speed/accuracy trade-offs in target-directed movements. Behavioral \& Brain Sciences, 20, 279-349.

Rosenbaum, D. A. (1980). Human movement initiation: Specification of arm, direction, and extent. Journal of Experimental Psychology: General, 109, 444-474.

Sirigu, A., Duhamel, J.-R., Cohen, L., Pillon, B., Dubois, B., \& AgID, Y. (1996). The mental representation of hand movements after parietal cortex damage. Science, 273, 1564-1568.

Soechting, J. F., \& Flanders, M. (1992). Moving in three-dimensional space: Frames of references, vectors, and coordinate systems. Annual Review of Neuroscience, 15, 167-191.

WELFORD, A. T. (1968). Fundamentals of skill. London: Methuen.

WoODWORTH, R. S. (1899). The accuracy of voluntary movement. Psychological Review Monographs, 3, 1-114.

(Manuscript received January 11, 2006; revision accepted for publication April 6, 2006.) 